\title{
Echocardiographic and Electrocardiographic Findings in COVID-19 Patients; A Cross-Sectional Study
}

Faeze Keihanian ( $\nabla$ faezekeihanian@yahoo.com )

Mashhad University of Medical Sciences

Hoorak Poorzand

Mashhad University of Medical Sciences

Amin Saeidinia

Mashhad University of Medical Sciences

Ali Eshraghi

Mashhad University of Medical Sciences

\section{Research Article}

Keywords: COVID-19, Echocardiography, Electrocardiography, Right Ventricular Dysfunction

Posted Date: January 3rd, 2022

DOI: https://doi.org/10.21203/rs.3.rs-1164425/v1

License: (a) (i) This work is licensed under a Creative Commons Attribution 4.0 International License.

Read Full License 


\section{Abstract}

Background: There are still many gaps in our knowledge regarding the direct cardiovascular injuries due to COVID-19 infection. In this study, we tried to find out the effect of SARS-CoV-2 infection on cardiac function in patients without any history of structural heart disease by electrocardiographic and echocardiographic evaluations.

Methods: This was a cross-sectional study on patients with COVID-19 infection admitted to Imam Reza hospital, Mashhad, Iran between 14 April and 21 September 2020. COVID-19 infection was verified by a positive reverse-transcriptase polymerase chain reaction (PCR) assay for SARS-CoV-2 using nasopharyngeal/oropharyngeal samples. We enrolled all patients over 18 years old with definite diagnosis of COVID-19 infection. All patients underwent a comprehensive transthoracic echocardiography at the first week of admission. Clinical and imaging data were collected prospectively.

Results: In total, 142 patients were enrolled in this study. The mean age of participants was $60.69 \pm 15.70$ years (range: $30-90$ years). Most patients were male (82, 57.7\%). Multivariate analysis showed that 02 saturation at admission was independently a predictor of re-hospitalization $(P<0.001)$. RV size $(P<0.001)$, dyslipidemia $(P<0.001)$, ejection fraction $(E F)(P<0.001)$, age $(P=0.020)$, systolic blood pressure $(P=0.001), 02$ saturation $(P=0.018)$ and diabetes $(P=0.025)$ independently predicted 30 -days mortality.

Conclusion: Echocardiography can be used for risk assessment in patients with COVID-19, especially in those with previous history of diabetes and dyslipidemia. The infection could result in Ventricular dysfunction, even in those without previous history of structural heart disease.

\section{Background}

Knowledge about short- and long-term complications of COVID-19 infection is growing worldwide 1 . COVID-19 is now a serious pandemic infection with different clinical features ${ }^{2}$. It is shown that severe acute respiratory syndrome coronavirus 2 (SARS-CoV-2) can involve respiratory tract and patients with previous cardiovascular disorders are at a higher risk ${ }^{3}$. The underlying mechanisms are yet to be clear ${ }^{4}$. It has been suggested that cardiac injury in COVID-19 infection is possibly related to its clinical outcomes. It was reported that patients with cardiac injury are associated with higher rate of morbidity, more abnormal laboratory and radiological findings ${ }^{5}$. Pathological findings (mononuclear inflammatory infiltration in heart tissue) in patients with COVID-19 infection suggests a direct damage to cardiac tissues ${ }^{6}$. Moreover, it is indicated that previous cardiovascular disorders may also be more predisposing to cardiac injury due to COVID-19 infection ${ }^{5}$. Keeping in mind the cardiovascular presentations, underlying cardiovascular disorders and its adverse events can help the clinician to make better decisions in the management of COVID-19 infection ${ }^{2}$.

Cardiac adverse events related to COVID-19 infection are prevalent (nearly 25\%) and can increase the risk of mortality ${ }^{7}$. Most recent studies on COVID-19 cardiac complications have reported clinical or laboratory 
findings with no imaging confirmation.

Transthoracic echocardiography is a reliable technique to detect ventricular function or valve disorders and risk assessment, as an additive guide to select better treatment strategies for hemodynamic stabilization ${ }^{8}$. However, due to a risk of viral contamination, echocardiographic evaluation in patients with COVID-19 infection has been limited 9, 10. To improve our knowledge about the pathophysiology of direct cardiovascular injuries due to COVID-19 infection ${ }^{2}$, we tried to find the effect of SARS-CoV-2 infection on cardiac function in patients without any history of structural heart disease by electrocardiographic and echocardiographic evaluations.

\section{Methods}

This was a cross-sectional study, performed on admitted patients with COVID-19 infection, in Imam Reza hospital, Mashhad, Iran between 14 April and 21 September 2020.

\subsubsection{Eligibility}

COVID-19 infection was verified by a positive reverse-transcriptase polymerase chain reaction (PCR) assay for SARS-CoV-2 using nasopharyngeal/oropharyngeal samples. We included all patients over 18 years old and a definite diagnosis of COVID-19 infection. We excluded patients with previous structural heart disease by history taking or evidences of old myocardial infarction in ECG. Figure1 shows the flowchart of study.

\subsubsection{Evaluations}

At admission, demographic data, comorbid conditions, medications, physical examination, lung CT scan and laboratory findings were all recorded. Cardiac injury was determined as troponin I (TPI) above the 99th percentile at presentation. Clinical and imaging data were collected prospectively and a 12-lead electrocardiography was performed. All patients were followed about three months for re-admission, cardiac events and in-hospital mortality or mortality.

\subsubsection{Echocardiography}

All patients underwent a comprehensive transthoracic echocardiography (TTE) within two weeks after symptoms presentation, in a standard manner with the same equipment (M7, Mindray Bio-Medical Electronics, Shenzhen, China) and by one cardiologist. All echocardiographic studies were performed bedside at COVID-19 intensive care unit (ICU) or non-ICU. Personal protection included airborne precautions, N-95 respirator masks, fluid-resistant gowns, and 2 sets of gloves, head covers, eye shields, and shoe covers. Most of measurements were performed offline to reduce the exposure time and contamination with two-to-three-seconds clips derived from standard echo views. ventricular size, systolic and diastolic function, pulmonary artery systolic pressure (PASP), presence of pericardial effusion, valvular stenosis or regurgitation were assessed. Abnormal TTE was defined as LV and RV enlargement, 
RV dysfunction (by visual assessment), ejection fraction (EF) $\leq 54 \%$, pericardial effusion, $P A S P \geq 35$ $\mathrm{mmHg}$, moderate and severe diastolic dysfunction and valvular regurgitation or stenosis more than mild.

\subsubsection{Exclusion criteria}

In order to better assessment of direct effects of COVID-19 on the echocardiographic parameters, patients with documented history of previous structural heart diseases (like valvular stenosis, primary valvular regurgitation, previous $\mathrm{Ml}$ and heart surgery, etc.) were excluded.

\subsubsection{Ethics}

The ethics committee of Mashhad University of Medical Sciences approved the study (Ethical No IR.MUMS.REC.1399.016). We obtained an informed consent from all patients or their legal guardians. The study was conducted in accordance with the declaration of Helsinki. Informed written consent was given prior to the inclusion of subjects or their families, in the study.

\section{Results}

In Total, 142 patients with diagnosis of Covid-19 infection, were enrolled who were admitted in Imam Reza Hospital, Mashhad, Iran. The mean age of participants was $60.69 \pm 15.70$ years (range: $30-90$ ). Most patients were male $(82,57.7 \%)$. Known comorbidities were found in $45.1 \%$, with hypertension $(24.6 \%)$ and diabetes mellitus $(23.9 \%)$ as the most common ones. The more frequent symptoms were dyspnea on admission (90.1\%), cough (54.9\%) and fever (49.3\%). Baseline demographic characteristics, comorbidities, clinical and laboratory data were presented in Table 1.

LAD was the most common finding (15.5\%) in ECG. Seventy-eight patients had sinus tachycardia (55\%), one atrial fibrillation ( $0.7 \%$ ) and two AVNRT (1.4\%). Ten patients had right axis deviation (RAD) and others normal axis.

Bilateral peripheral ground glass opacification (GGO) was the most common specific CT scan manifestation (38\%) followed by diffused bilateral GGO (35.2\%), unilateral GGO (14.1\%), and bilateral pleural effusion (9.9\%). Other non-specific presentations were consolidation (63.4\%), and lymphadenopathy (31\%).

Abnormal TTE was seen in 71 cases (50\%). Preserved left ventricle EF ( $E F=50-54 \%)$ was noted in 17 cases and reduced $\mathrm{EF}(\mathrm{EF}<50 \%)$ in $8(5.6 \%)$. Seventy-nine patients had mild diastolic dysfunction (56.3\%), two of them moderate (1.4\%) and others had normal diastolic function. Mild RV dilation was seen in 14 cases (9.9\%) and moderate in two cases (1.4\%). Mild pulmonary hypertension (PHT) was found in 12 subjects. Representative example of mild RV enlargement and mild PHT in a COVID-19 patient without prior cardiovascular disorder is shown in Figure2. Pericardial effusion was detected in 32 cases (22 minimal PE and 10 mild PE). The echocardiographic indices are shown in Table 2.

RV dysfunction was reported in 49 patients (34.5\%) (39 mild RV dysfunction, 8 moderate, 2 severe). Table 3 demonstrates the patients' characteristics stratified by RV function. RV size was correlated with 02 
saturation ( $r=-0.302, P<0.001), \operatorname{TPI}(r=0.337, P=0.019), E S R(r=0.207, P=0.035)$ and Platelet count $(r=$ $0.355, P<0.001)$. CPK $(P=0.033)$ and calcium $(P=0.005)$ levels were significantly higher in patients with RV dysfunction.

Our findings demonstrated that SBP (OR: 0.885, $P=0.006)$, DBP (OR: 1.24, $P=0.003)$, male gender (OR: $0.40, P=0.004$ ), higher age (OR: 1.37, $P=0.002$ ), smoking (OR: 0.011, $P=0.035$ ), addiction (OR: 0.011, $\mathrm{P}=0.011$ ), hypertension (OR: $2.41, \mathrm{P}=0.003$ ), asthma (OR: $0.10, \mathrm{P}=0.032$ ), bilateral peripheral $\mathrm{GGO}$ (OR: $23.6, P=0.019)$ and coronary calcification (OR: 5.74, $P=0.005)$ were predictors of in-hospital mortality. Moreover, age (OR: 1.29, $\mathrm{P}=0.002$ ), hypertension (OR: 2.99, $\mathrm{P}=0.004)$ and bilateral peripheral $\mathrm{GGO}(\mathrm{OR}$ : $26.81, \mathrm{P}=0.023)$ were predictors of 30-day mortality.

In multivariate regression analysis, lower 02 saturation at the time of admission was independently predictor of re-admission $(P<0.001)$. Furthermore, PASP $(P=0.026)$, dyslipidemia $(P=0.002)$ and $R V$ dilation $(P=0.037)$ were significantly predictors of in-hospital mortality (after adjusting for possible cofounders). RV enlargement $(P<0.001)$, dyslipidemia $(P<0.001)$, lower LVEF $(P<0.001)$, older age $(P=0.020), \operatorname{SBP}(P=0.001), 02$ saturation $(P=0.018)$, shorter pulmonary acceleration time (PAT) $(P=0.005)$ and diabetes $(P=0.025)$ independently predicted 30 -days mortality.

Patients with cardiac injury were mostly females $(P=0.001)$, had hypertension $(P=0.001)$, more comorbidities ( $P=0.026)$, bilateral peripheral $G G O(P=0.001)$, RV dysfunction $(P=0.029)$, a higher level of CPK $(P=0.018)$ and coronary calcification $(P=0.006)$, compared to those without. Likewise, in patients with cardiac injury, pericardial effusion was significantly more reported (16 versus one, $P=0.021)$.

LV dysfunction (EF lesser than $55 \%$ ) was seen in $17.6 \%$ of cases; probably most of them due to COVID-19 related cardiac injury.

\section{Discussion}

Investigations regarding cardiovascular complications in COVID-19 in developing countries are limited. To the best of our knowledge, our study is the first to assess cardiac function in COVID-19 patients using both echocardiography and ECG findings in those without any significant structural heart diseases. Our aim was to evaluate the direct impact of SARS-CoV-2 infection on cardiovascular indices in hospitalized patients.

As above-mentioned, patients with cardiac injury had a higher level of CPK, comorbidities and RV dysfunction. In a similar study, patients with cardiac injury had higher levels of inflammatory biomarkers, underwent mechanical ventilation more probably and had higher mortality rate and RV dysfunction. They showed that two indices of strain echocardiography, i.e. LV global longitudinal strain and RV FWLS were are powerful predictors of higher mortality in patients with COVID-19 infection ${ }^{11}$.

RV dysfunction in COVID-19 patients is common and associated with a pro-thrombotic and inflammatory state which is determined by D-dimer and CRP elevation. In our study, RV systolic dysfunction was 
reported in 49 cases (34.5\%) and LV systolic dysfunction in 17.6\%. We showed a significant correlation between RV size and TPI level. In another retrospective study, LV systolic function was hyper-dynamic or normal in most cases (89\%), RV was dilated in $41 \%$, and impaired in $27 \%$. It was demonstrated that RV systolic dysfunction (defined by fractional area change) was significantly associated with elevated Ddimer and CRP but not related to hsTnl ${ }^{9}$.

Similar to our study, in another investigation, all indices of RV hemodynamics and function were worse compared to the LV parameters. They introduced RV dilation and dysfunction as major abnormal echocardiographic patterns among deteriorating patients ${ }^{10}$. Many conditions can increase pulmonary vascular resistance or pulmonary pressure in hospitalized patients and precipitate acute RV failure. These conditions include pulmonary embolism (PE), hypoxic pulmonary vasoconstriction, decrease in lung volume, excessive positive end-expiratory pressure, pneumonia, hyper-carbia, the use of a-agonists or a combination of all these factors ${ }^{10}$. In COVID-19 infection, the probable mechanisms capable for this condition include: 1 ) direct myocardial injury by virus; 2 ) increased RV afterload in ARDS; 3) hypoxic pulmonary vasoconstriction; 4) pulmonary micro-thrombosis, and 5) endothelial and microvascular injury 12. Besides, RV dilation and dysfunction may also affect LV function by ventricular interdependence and paradoxical septal motion which causes reduction in LV volume and cardiac output ${ }^{11}$. We showed that RV dilation is a predictor of in-hospital and 30-days mortality in COVID-19 patients without any previous structural heart diseases. Similarly, Argulian et al. showed that RV dilation was predictive for in-hospital mortality in patients with COVID-19 ${ }^{13}$. Another observation was reported by Szekely et al. ${ }^{10}$, who demonstrated that increased RV end diastolic area was significantly associated with mortality. We demonstrated that dyslipidemia, lower EF, older age, SBP, 02 saturation, PAT and diabetes were also independently predictors of 30-days mortality. In line with our study, the only baseline echocardiographic parameters significantly associated with mortality were low LVEF, elevated E/e' ratio, increased RV end diastolic area, and higher Tei index ${ }^{10}$. Therefore, identifying RV impairment would guide physicians to limit positive end-expiratory pressure and avoid hyper-capnic state, which could adversely affect RV performance by inducing pulmonary arteriolar vasoconstriction and increased RV afterload ${ }^{9}$.

During acute RV pressure overload and resultant RV dysfunction and reduced cardiac output, systemic blood pressure will decrease, which may result in lowering coronary perfusion and additional reduction in RV contractility ${ }^{10}$.

In Szekely et al study, patients with shorter PAT were older, had more comorbidities, and worse lung disease, lower oxygen saturation, higher LV filling pressure, and higher biomarkers (D-dimer, BNP, Troponin-I, and CRP). They suggested that elevated pulmonary vascular resistance in COVID-19 infection is multifactorial and related to parenchymal lung disease, pulmonary vascular disease, and elevated left atrial pressure, all leading to cardiac injury ${ }^{10}$. Similar to our findings, there is a low prevalence of LV impairment in prior studies ${ }^{9}$. Previous case reports and publications showed that COVID-19 is a known cause of severe cardiac dysfunction such as fulminant myocarditis, even without common COVID-19 
symptoms or pulmonary involvement ${ }^{14-16}$. Cardiac injury is prevalent in patients admitted with COVID19 infection and related to high mortality rate ${ }^{2}$ but LV systolic dysfunction is not very common.

This study performed on a larger sample size compared to previous investigations. We enrolled only patients with positive PCR test for COVID-19. Another strength of this study was excluding patients with previous underlying heart diseases, which helps to elucidate the pure effects of COVID-19 on cardiovascular system. Besides, all of evaluations were performed by a single cardiologist, which eliminates inter-observer discrepancies.

Limitation:

Further studies on the reasons of RV dysfunction are needed.

\section{Conclusion}

RV systolic dysfunction was prevalent in patients with COVID-19 and can be correlated with higher mortality. Moreover, lower LVEF, shorter PAT and lower 02 saturation, older age, elevated SBP, dyslipidemia and diabetes were independently predictors of 30-days mortality. Using echocardiography for risk assessment in patients with COVID-19, especially with previous history of diabetes, hypertension and dyslipidemia, can be helpful and lead to better management.

\section{Abbreviations}

COVID-19

Coronavirus disease 2019

SARS-CoV-2

Severe acute respiratory syndrome coronavirus 2

PCR

polymerase chain reaction

CT

computerized tomography

ECG

Electrocardiography

RV

Right ventricle

PASP

pulmonary artery systolic pressure

$\mathrm{EF}$

ejection fraction

TPI

troponin I 
TTE

transthoracic echocardiography

GGO

ground glass opacification

PHT

pulmonary hypertension

ESR

Erythrocyte Sedimentation Rate

CPK

Creatine phosphokinase

SBP

systolic blood pressure

DBP

diastolic blood pressure

OR

Odds ratio

PAT

pulmonary acceleration time

FWLS

Free wall longitudinal strain

CRP

$\mathrm{C}$ reactive protein

hsTnl

high sensitive troponin I

PE

pulmonary embolism

ARDS

Acute respiratory distress syndrome

BNP

Brain natriuretic peptide

\section{Declarations}

\section{Funding}

This study was funded by Mashhad University of Medical Sciences.

\section{Availability of data and materials}

The datasets used or analyzed in the current study are available from the corresponding author on reasonable request. 


\section{Ethics approval and consent to participate}

This study was approved by the ethical committee of the medical faculty of Mashhad University of Medical Sciences. The ethical code of the study was IR.MUMS.REC.1399.016. All patients filled a written informed consent form before entering the study.

\section{Competing interests}

The authors declare that they have no competing interests.

\section{Authors' contributions}

Dr. Faeze Keihanian designed the study, collected data and performed echocardiographic assessments, wrote the first draft of the article and approved the final version; Dr. Hoorak Poorzand participated in data analysis and echocardiographic assessments, read the final version and approved it; Dr. Amin Saeidinia participated in data collection, statistical analysis and writing first draft of the article. Dr. Ali Eshrgahi designed the study, wrote the first draft of the article and approved the final version.

\section{Acknowledgement}

We would like to thank the deputy of research, Mashhad University of Medical Sciences and physicians and nurses working hard in COVID-19 wards in Imam Reza hospital.

\section{Consent for publication}

All patients took consent to publish their data anonymously.

\section{References}

1. NeJhaddadgar N, Ziapour A, Zakkipour G, Abbas J, Abolfathi M, Shabani M Effectiveness of telephone-based screening and triage during COVID-19 outbreak in the promoted primary healthcare system: a case study in Ardabil province, Iran. Journal of Public Health2020:1-6

2. Keihanian F, Bigdelu L (2020) Cardiovascular Considerations in COVID19: A Comprehensive Review. Ther Clin Risk Manag 16:1089-1097

3. Force ADT (2012) Acute respiratory distress syndrome. JAMA 307:2526-2533

4. Zhou F, Yu T, Du R, Fan G, Liu Y, Liu Z, Xiang J, Wang Y, Song B, Gu X (2020) Clinical course and risk factors for mortality of adult inpatients with COVID-19 in Wuhan, China: a retrospective cohort study. The lancet 
5. Shi S, Qin M, Shen B, Cai Y, Liu T, Yang F, Gong W, Liu X, Liang J, Zhao Q, Huang H, Yang B, Huang C (2020) Association of Cardiac Injury With Mortality in Hospitalized Patients With COVID-19 in Wuhan, China. JAMA Cardiology 5:802-810

6. Xu Z, Shi L, Wang Y, Zhang J, Huang L, Zhang C, Liu S, Zhao P, Liu H, Zhu L (2020) Pathological findings of COVID-19 associated with acute respiratory distress syndrome. The Lancet respiratory medicine 8:420-422

7. Guo T, Fan Y, Chen M, Wu X, Zhang L, He T, Wang H, Wan J, Wang X, Lu Z (2020) Cardiovascular implications of fatal outcomes of patients with coronavirus disease 2019 (COVID-19). JAMA cardiology

8. Mitchell M, Fonarow J, Peterson G, Geraci P, Horwich S (2013) T. 2013 ACCF/AHA Guideline for the Management of Heart Failure. Executive Summary

9. Mahmoud-Elsayed HM, Moody WE, Bradlow WM, Khan-Kheil AM, Senior J, Hudsmith LE, Steeds RP (2020) Echocardiographic Findings in Patients With COVID-19 Pneumonia. Can J Cardiol 36:12031207

10. Szekely Y, Lichter Y, Taieb P, Banai A, Hochstadt A, Merdler I, Gal Oz A, Rothschild E, Baruch G, Peri Y (2020) The Spectrum of Cardiac Manifestations in Coronavirus Disease 2019 (COVID-19)-a Systematic Echocardiographic Study. Circulation

11. Xie Y, Wang L, Li M, Li H, Zhu S, Wang B, He L, Zhang D, Zhang Y, Yuan H (2020) Biventricular Longitudinal Strain Predict Mortality in COVID-19 Patients. Frontiers in cardiovascular medicine 7:418

12. Park JF, Banerjee S, Umar S (2020) In the eye of the storm: the right ventricle in COVID-19. Pulmonary Circulation 10:2045894020936660

13. Argulian E, Sud K, Vogel B, Bohra C, Garg VP, Talebi S, Lerakis S, Narula J (2020) Right ventricular dilation in hospitalized patients with COVID-19 infection. Cardiovascular Imaging 13:2459-2461

14. Paul J-F, Charles P, Richaud C, Caussin C, Diakov C (2020) Myocarditis revealing COVID-19 infection in a young patient. European Heart Journal - Cardiovascular Imaging 21:776-776

15. Beşler MS, Arslan H (2020) Acute myocarditis associated with COVID-19 infection. The American Journal of Emergency Medicine;38:2489.e2481-2489.e2482

16. Doyen D, Moceri P, Ducreux D, Dellamonica J (2020) Myocarditis in a patient with COVID-19: a cause of raised troponin and ECG changes. The Lancet 395:1516

\section{Tables}

Table 1-Baseline demographic characteristics, comorbidities, clinical and laboratory data 


\section{Parameter}

\begin{tabular}{|c|c|}
\hline Age (years) (mean $\pm S D)$ & $60.69 \pm 15.70$ \\
\hline \multicolumn{2}{|l|}{$\operatorname{Sex}(N, \%)$} \\
\hline Male & $82,57.7$ \\
\hline Female & $60,42.3$ \\
\hline \multicolumn{2}{|l|}{ Comorbidities (N, \%) } \\
\hline Diabetes & $34,23.9$ \\
\hline Hypertension & $34,23.9$ \\
\hline Dyslipidemia & $16,11.3$ \\
\hline History of Stroke & $4,2.8$ \\
\hline Chronic kidney disease & $6,4.2$ \\
\hline Lung disease & $9,6.3$ \\
\hline History of malignancy & $3,2.1$ \\
\hline Current smoker & $8,5.6$ \\
\hline Addiction & $17,11.9$ \\
\hline \multicolumn{2}{|l|}{ Vital sign (Mean \pm S.D) } \\
\hline Heart rate (beats/minute) & $102.43 \pm 19.23$ \\
\hline Systolic blood pressure (mmHg) & $135.46 \pm 20.66$ \\
\hline Diastolic blood pressure $(\mathrm{mmHg})$ & $84.62 \pm 20.66$ \\
\hline $\mathrm{O}_{2}$ saturation $(\%)$ & $88.31 \pm 7.83$ \\
\hline Temperature $\left({ }^{\circ} \mathrm{C}\right)$ & $37.38 \pm 0.79$ \\
\hline Respiratory rate (breaths/minute) & $23.92 \pm 5.42$ \\
\hline \multicolumn{2}{|l|}{ Symptoms (N, \%) } \\
\hline Cough & $78,54.9$ \\
\hline Dyspnea & $129,90.8$ \\
\hline Fever & $70,49.3$ \\
\hline Weakness & $57,40.1$ \\
\hline Chest pain & $2,1.4$ \\
\hline Gastrointestinal & $25,17.6$ \\
\hline Confusion & $8,5.6$ \\
\hline
\end{tabular}




\begin{tabular}{ll} 
Laboratory data (Mean \pm S.D) & \\
Blood sugar (mg/dL) & $142.12 \pm 22.88$ \\
Urea (mg/dL) & $43.94 \pm 17.03$ \\
Creatinine (mg/dL) & $1.34 \pm 0.7$ \\
Sodium (meq/L) & $136.28 \pm 3.49$ \\
Potassium (meq/L) & $4.09 \pm 0.60$ \\
Total bilirubin (mg/dL) & $0.61 \pm 0.43$ \\
Direct bilirubin (mg/dL) & $0.25 \pm 0.15$ \\
Calcium (mg/dL) & $8.54 \pm 0.89$ \\
Magnesium (mg/dL) & $2.06 \pm 0.42$ \\
Aspartate transaminase (IU/L) & $44.90 \pm 18.23$ \\
Alanine aminotransferase (IU/L) & $49.06 \pm 17.66$ \\
Alkaline phosphatase (IU/L) & $212.32 \pm 80.91$ \\
Lactate dehydrogenase (IU/L) & $606.65 \pm 230.51$ \\
Creatine phosphokinase (IU/L) & $350.97 \pm 143.38$ \\
Cardiac troponin I (ng/ml) & $56.22 \pm 25.24$ \\
PCO2 (mmHg) & $42.10 \pm 13.79$ \\
HCO3 (meq/L) & $27.28 \pm 6.57$ \\
White blood cell (10\%/L) & $8860.56 \pm 5732.48$ \\
PMN (\%) & $77.88 \pm 9.31$ \\
Lymph (\%) & $16.65 \pm 7.93$ \\
Hemoglobin (g/dL) & $13.18 \pm 2.11$ \\
MCV (fL) & $84.54 \pm 7.01$ \\
RDW (\%) & $14.08 \pm 1.74$ \\
CRP (mg/elet (10 $9 / \mathrm{L})$ & $222.18 \pm 126.55$ \\
ESR (mm/h) & $88.63 \pm 74.66$ \\
\hline
\end{tabular}

PCO2= Partial pressure of carbon dioxide; $H C O 3=$ Bicarbonate $P M N=$ Polymorphonuclear leukocytes; $L y m p h=L y m p h o c y t e ; M C V=$ Mean corpuscular volume; $R D W=$ Red cell distribution width; $C R P=C$-reactive 
protein; ESR = Erythrocyte sedimentation rate

Table 2-Echocardiographic findings of patients

\begin{tabular}{|ll|}
\hline Echocardiographic parameters & Mean \pm S.D \\
\hline LVEDD & $42.08 \pm 4.70$ \\
\hline LVEF & $54.96 \pm 4.23$ \\
\hline E & $74.47 \pm 18.82$ \\
\hline A & $84.67 \pm 22.01$ \\
\hline E' $^{\prime}$ & $9.16 \pm 2.39$ \\
\hline E/ ' & $7.95 \pm 2.34$ \\
\hline LVOT VTI & $18.87 \pm 3.98$ \\
\hline TV annulus & $29.10 \pm 4.12$ \\
\hline RV diameter & $27.27 \pm 3.97$ \\
\hline RV S' & $12.33 \pm 2.36$ \\
\hline TAPSE & $19.04 \pm 3.39$ \\
\hline RVOT VTI & $16.65 \pm 3.24$ \\
\hline PAT & $97.63 \pm 22.32$ \\
\hline PASP & $24.43 \pm 6.52$ \\
\hline
\end{tabular}

$\angle V E D D=L$ eft ventricular end diastolic diameter; $\angle V E F=L$ eft ventricular ejection fraction; $\angle V O T=L$ eft ventricular outflow tract; VTI= Velocity Time Integral; RV = Right ventricle; TAPSE = Tricuspid annular plane systolic excursion; $R V O T=$ Right ventricular outflow tract; $P A T=$ Pulmonary acceleration time; $P A S P=$ Pulmonary artery systolic pressure

Table 3- Patients' characteristics Stratified by RV function 


\begin{tabular}{|c|c|c|c|c|}
\hline \multicolumn{2}{|l|}{ Parameter } & $\begin{array}{l}\text { RV dysfunction } \\
(n=50)\end{array}$ & $\begin{array}{l}\text { Normal RV } \\
\text { function } \\
(n=92)\end{array}$ & P Value \\
\hline \multicolumn{2}{|l|}{ Age (Mean \pm S.D) } & $62.63 \pm 16.29$ & $59.26 \pm 15.63$ & 0.240 \\
\hline \multirow[t]{2}{*}{ Gender (N, \%) } & Male & $23,46.9$ & $59,63.4$ & \multirow[t]{2}{*}{0.044} \\
\hline & Female & $26,53.1$ & $34,36.6$ & \\
\hline \multicolumn{2}{|c|}{ Heart Rate (Mean \pm S.D) } & $100.63 \pm 19.69$ & $103.24 \pm 19.21$ & 0.464 \\
\hline \multicolumn{2}{|c|}{ Systolic Blood Pressure (Mean \pm S.D) } & $135.40 \pm 19.86$ & $135.39 \pm 21.45$ & 0.999 \\
\hline \multicolumn{2}{|c|}{ Diastolic Blood Pressure (Mean \pm S.D) } & $83.73 \pm 12.77$ & $85.48 \pm 16.01$ & 0.504 \\
\hline \multicolumn{2}{|c|}{$\mathrm{O}_{2}$ saturation (Mean $\pm \mathrm{S} . \mathrm{D}$ ) } & $88.10 \pm 5.88$ & $88.42 \pm 8.85$ & 0.808 \\
\hline \multicolumn{5}{|c|}{ Laboratory data (Mean \pm S.D) } \\
\hline \multicolumn{2}{|l|}{ Urea } & $41.18 \pm 16.77$ & $44.46 \pm 17.37$ & 0.556 \\
\hline \multicolumn{2}{|l|}{ Creatinine } & $1.23 \pm 0.7$ & $1.40 \pm 0.8$ & 0.595 \\
\hline \multicolumn{2}{|l|}{ Calcium } & $8.87 \pm 0.99$ & $8.35 \pm 0.78$ & 0.005 \\
\hline \multicolumn{2}{|c|}{ Lactate dehydrogenase } & $598.51 \pm 211.97$ & $606.82 \pm 241.96$ & 0.835 \\
\hline \multicolumn{2}{|c|}{ Creatine phosphokinase } & $472.00 \pm 181.02$ & $243.50 \pm 146.01$ & 0.033 \\
\hline \multicolumn{2}{|l|}{ White blood cell } & $\begin{array}{l}7302.04 \pm \\
3021.20\end{array}$ & $9621.97 \pm 6665.41$ & 0.064 \\
\hline \multicolumn{2}{|l|}{ Hemoglobin } & $13.58 \pm 2.16$ & $12.92 \pm 2.06$ & 0.108 \\
\hline \multicolumn{2}{|l|}{ RDW } & $14.45 \pm 1.96$ & $13.84 \pm 1.57$ & 0.082 \\
\hline \multicolumn{2}{|l|}{ PMN } & $76.03 \pm 8.69$ & $78.76 \pm 9.61$ & 0.985 \\
\hline \multicolumn{2}{|l|}{ Lymph } & $18.16 \pm 7.04$ & $15.87 \pm 8.37$ & 0.090 \\
\hline \multicolumn{5}{|l|}{ Platelet } \\
\hline \multicolumn{2}{|l|}{ CRP } & $80.44 \pm 63.8$ & $92.69 \pm 79.49$ & 0.370 \\
\hline \multicolumn{2}{|l|}{ ESR } & $47.25 \pm 30.33$ & $39.47 \pm 30.10$ & 0.217 \\
\hline \multicolumn{5}{|l|}{ ECG assessment } \\
\hline \multirow[t]{3}{*}{ ECG Rhythm (\%) } & Sinus Tachycardia & 48.9 & 49.4 & \multirow[t]{3}{*}{0.740} \\
\hline & Atrial Fibrillation & 0 & 1.3 & \\
\hline & Normal sinus rhythm & 51.1 & 49.4 & \\
\hline ECG Axis (\%) & Normal axis & 73.3 & 74.7 & 0.543 \\
\hline
\end{tabular}




\begin{tabular}{|c|c|c|c|c|}
\hline & LAD & 22.2 & 15.6 & \\
\hline & RAD & 4.4 & 10.1 & \\
\hline ECG abnormality & RBBB & 8.2 & 6.6 & 0.42 \\
\hline & LAHB & 12.2 & 9.9 & \\
\hline & LBBB & 4.1 & 0 & \\
\hline & $\mathrm{LAHB}+\mathrm{RBBB}$ & 4.1 & 0 & \\
\hline Symptoms (\%) & & & & \\
\hline Chest Pain & & 4.1 & 0 & 0.121 \\
\hline Nausea and vomi & & 12.2 & 13.2 & 0.550 \\
\hline Diarrhea & & 8.2 & 2.2 & 0.112 \\
\hline Dyspnea & & 87.8 & 91.2 & 0.354 \\
\hline Cough & & 55.1 & 56 & 0.528 \\
\hline Fever & & 51 & 49.5 & 0.50 \\
\hline Weakness & & 26.5 & 45.1 & 0.024 \\
\hline Confusion & & 8.2 & 4.4 & 0.289 \\
\hline Comorbidities (\%) & & & & \\
\hline Diabetes & & 28.6 & 22 & 0.253 \\
\hline Addiction & & 5.6 & 6.3 & 0.667 \\
\hline Smoker & & 2.1 & 3.5 & 0.580 \\
\hline Dyslipidemia & & 12.2 & 11 & 0.513 \\
\hline Hypertension & & 20.4 & 26.4 & 0.284 \\
\hline History of stroke & & 0 & 4.4 & 0.174 \\
\hline Asthma & & 4.1 & 6.6 & 0.424 \\
\hline COPD & & 0 & 2.2 & 0.421 \\
\hline Malignancy & & 2.2 & 4.1 & 0.437 \\
\hline ESRD & & 4.1 & 3.3 & 0.429 \\
\hline CT scan findings & & & & \\
\hline Bilateral peripher & & 46.9 & 34.1 & 0.095 \\
\hline Bilateral diffused & & 32.7 & 34.7 & 0.358 \\
\hline
\end{tabular}




\begin{tabular}{|c|c|c|c|c|}
\hline \multicolumn{2}{|l|}{ Unilateral GGO } & 8.2 & 17.6 & 0.10 \\
\hline \multicolumn{2}{|c|}{ Bilateral Pleural effusion } & 8.2 & 11 & 0.416 \\
\hline \multicolumn{2}{|l|}{ Consolidation } & 69.4 & 59.3 & 0.161 \\
\hline \multicolumn{2}{|c|}{ Lymphadenopathy } & 24.5 & 33 & 0.198 \\
\hline \multicolumn{5}{|c|}{ Echocardiographic parameters } \\
\hline \multicolumn{2}{|c|}{ LVEDD (Mean \pm S.D) } & $41.61 \pm 4.64$ & $42.27 \pm 4.77$ & 0.089 \\
\hline \multicolumn{2}{|c|}{ E/ e' (Mean \pm S.D) } & $8.05 \pm 3.09$ & $7.90 \pm 1.85$ & 0.755 \\
\hline \multirow[t]{3}{*}{ LVEF $(n, \%)$} & $\geq 55$ & $34,69.4$ & $79,84.9$ & \multirow[t]{3}{*}{0.024} \\
\hline & $50-54$ & $9,18.4$ & $12,12.9$ & \\
\hline & $40-49$ & $6,12.2$ & $2,2.2$ & \\
\hline \multicolumn{2}{|c|}{ Pulmonary hypertension (n, \%) } & $6,12.8$ & $5,5.6$ & 0.193 \\
\hline \multirow[t]{3}{*}{ RV size (n, \%) } & Normal & $41,83.7$ & $85,90.4$ & \multirow[t]{3}{*}{0.145} \\
\hline & Mild enlargement & $6,12.2$ & $8,8.6$ & \\
\hline & $\begin{array}{l}\text { Moderate } \\
\text { enlargement }\end{array}$ & $2,1.4$ & 0 & \\
\hline
\end{tabular}

$R D W=$ Red cell distribution width; $P M N=$ Polymorphonuclear leukocytes; Lymph= Lymphocyte; $C R P=C$ reactive protein; $E S R=$ Erythrocyte sedimentation rate; $E C G=$ Electrocardiogram; $L A D=L$ eft axis deviation; $R A D=$ Right axis deviation; $R B B B=$ Right bundle branch block; $\angle B B B=$ Left bundle branch block; $\angle A H B=$ Left anterior hemi block; $C O P D=$ Chronic obstructive pulmonary disease; $E S R D=$ End stage renal disease; $G G O=$ ground glass opacification; $L V E D D$ : Left ventricular end diastolic diameter; $L V E F=$ Left ventricular ejection fraction; $R V=$ Right ventricle

\section{Figures}




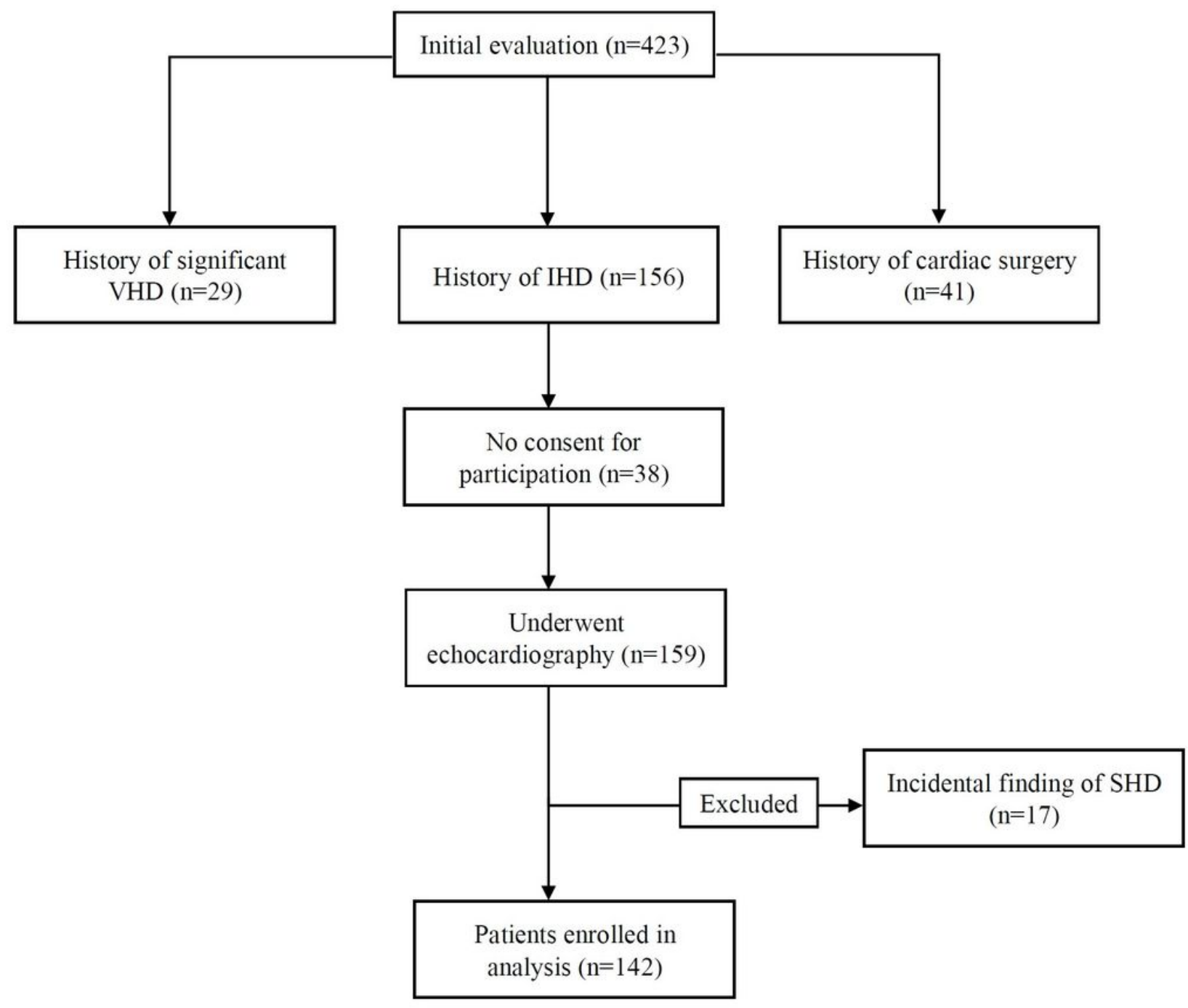

\section{Figure 1}

Flowchart of study. VHD= valvular heart disease; IHD= Ischemic heart disease; $S H D=$ structural heart disease.
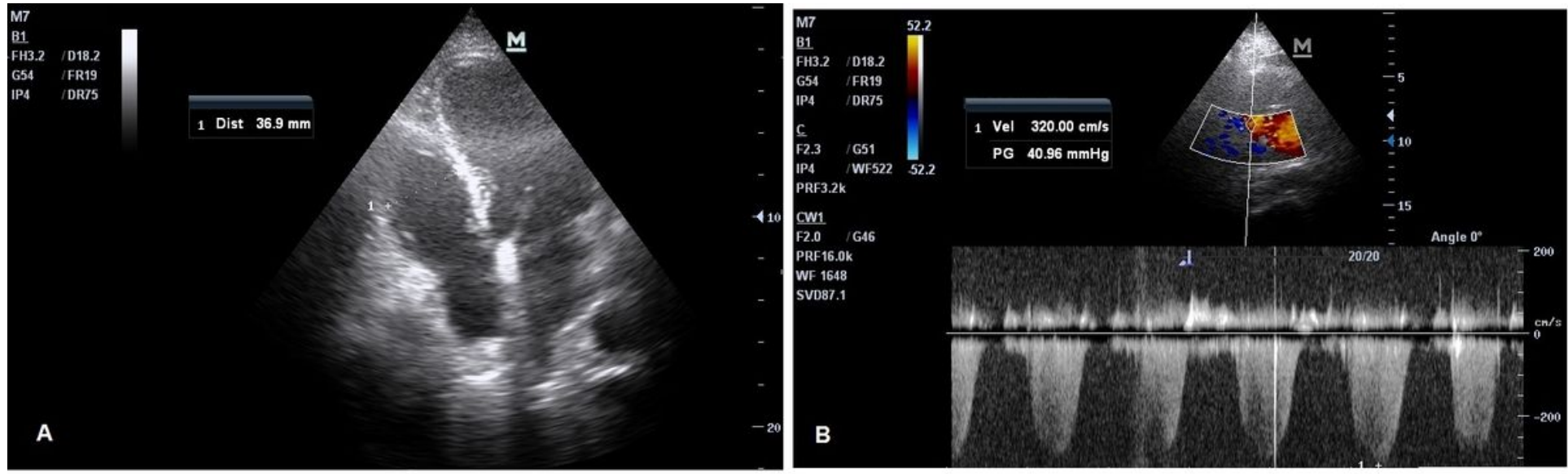

Figure 2 
Example of two-dimensional transthoracic echocardiography of a patient without previous cardiovascular disorder. A) Mild right ventricular enlargement in RV focused view; B) Off axis fourchamber view of the heart suggesting moderate TR with TR gradient about $41 \mathrm{mmHg}$. Estimated pulmonary arterial pressure in this patient was $44 \mathrm{mmHg}$ (considering normal IVC size and collapsibility). 